\title{
Cascades Neural Network based Segmentation of Fluorescence Microscopy Cell Nuclei
}

\author{
Sofyan M. A. Hayajneh*, Mohammad H. Alomari ${ }^{\dagger}$, Bassam Al-Shargabi ${ }^{*}$ \\ *Department of Electrical and Computer Engineering \\ Isra University, Amman, Jordan \\ ${ }^{\dagger}$ Department of Electrical Engineering \\ Applied Science Private University, Amman, Jordan \\ *Department of Computer Information System \\ Isra University, Amman, Jordan
}

\begin{abstract}
The visual extraction of cellular, nuclear and tissue components from medical images is very vital in the diagnosis routine of different health related abnormalities and diseases. The objective of this work is to modify and efficiently combine different image processing methods supported by cascaded artificial neural networks in an automated system to perform segmentation analysis of medical microscopy images to extract nuclei located in either simple or complex clusters. The proposed system is applied on a publicly available data sets of microscopy nuclei cells. A GUI is designed and presented in this work to ease the analysis and screening of these images. The proposed system shows promising performance and reduced computational time cost. It is hoped that thus system and the corresponding GUI will construct platform base for several biomedical studies in the field of cellular imaging where further complex investigations and modelling of microscopy images could take place.
\end{abstract}

Keywords-Artificial neural networks; machine learning, DSP, fluorescence microscopy; biomedical imaging; cell nuclei; image segmentation

\section{INTRODUCTION}

\section{A. Digital Image Processing}

Digital image processing and analysis is among rapidly growing technologies. It encompasses a wide-ranging field of applications in our everyday life. Medical, industrial, text recognition, biometrics and graphics, are just examples of hundreds of possible applications of image processing. Although every single application needs a well-designed approach to parse and extract the required useful information and, most of these approaches can be categorized under a single or multiple major aspects that include but are not limited to: image visualization, sharpening, enhancement, recognition, verification, retrieval, segmentation and /or restoration, etc. [1]-[4].

Most of the image processing-based applications are assembled and designed by applying a schematic classical methodology that includes one or more of the following phases [1]. Image pre-processing and enhancement, Objects segmentation (extraction), Statistical features extraction, Objects (Candidates) selection and pruning, Objects postprocessing and (or) Features classification.
Segmentation is an important phase in image analysis where the image is divided into meaningful disjoint regions with similar properties, such as gray level, color, texture, brightness, contrast, etc. It is often one of the first and most difficult phases in image analysis. Due to its importance, a great variety of segmentation algorithms have been proposed to tackle a wide range of applications such as microscopy, biomedical engineering, biomedical imaging, bioinformatics and pattern recognition [5]-[9]. The segmentation process will be the focus of our attention in this work and more details about segmentation process will be highlighted in Section II.

\section{B. Medical Imaging and Computer Aided Diagnosis (CAD)}

Medical imaging techniques are usually implemented to greatly enhance the extraction process of numerical features that provide efficient and as sufficient as possible representation of medical signs features, activities or general regions of interest in the different types of medical images acquired by different types of medical instruments. These images along with the possible integration of advanced image processing techniques and medical and physiology concepts can positively improve the ability of knowledge extraction and increase the ability to screen and (or) predict diseases that may have serious impacts on our daily life [10]-[12].

The difficult medical diagnostic routine (time consuming and tedious process) can be improved by providing the specialists (e.g. radiologists, pathologists, biologists) with quantitative data and statistical measurements which are extracted from such medical images so that the visualized version is much more informative [1], [13]. The development in computational power (e.g. processor speed, RAM, graphical hardware) has driven the development of several image processing algorithms that have had a significant impact in several medical research and applications.

Recently, computer aided diagnosis (CAD) has quickly become a widespread and unescapable useful tool for diagnostic examinations in many daily routine works across a wide range of medical and clinical areas such as microscopy imaging, tissue culturing, cancer research, Confocal microscopy, heart diseases, brain tumors, blood diseases screening, etc. [14]-[18]. Such CAD (Fig. 1) systems have drawn a lot of attention because they can represents a second opinion for the specialist and they allow a large scale 
statistical evaluation besides the classical human screening evaluation [19], [20].

\section{Fluorescence Microscopy and Nuclei Image Segmentation}

In fluorescence microscopy, the object under consideration is itself the light source rather than a light reflector. This is due the fact that certain material absorbs light at one wavelength (excitation) and emits a detectable visible (i.e. longer specific wavelength) light. This physical phenomenon (fluorescence) is utilized to visually and separately observe different components of the specimen such as cell membrane, cytoplasm, nuclei, gene, tissue, or proteins (Fig. 2).

The component of interest is specifically stained in the specimen using particular fluorescent dyes and then the fluorescence microscopes make it possible to visualize and store digital images of this component [21]. In fluorescence microscopy applications, researchers are typically interested in as much as accurate localization of the boundaries of the observed fluorescently labelled structural and functional units (cell nuclei, genes, etc.).

Heterogeneity of different biological features can be an issue arising from the use of fluorescence imaging data. However, to a large extent this may be overcome by specially designed CADs, which can correctly take into account the physical variations seen between cells and therefore across a set of fluorescence microscopy images.
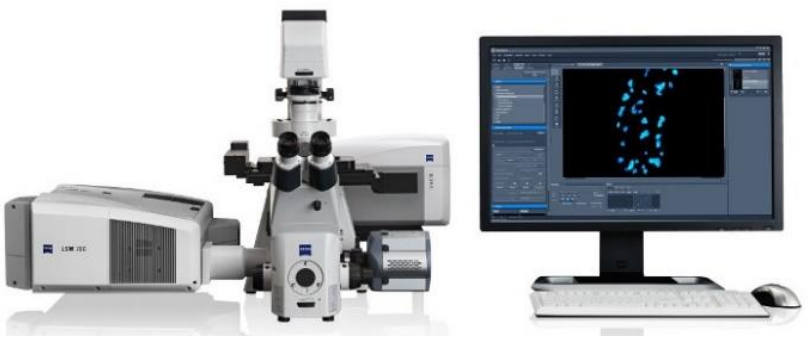

Fig. 1. Example of a CAD microscope (Image courtesy: Carl Zeiss- ELYRA Super resolution Microscopy).

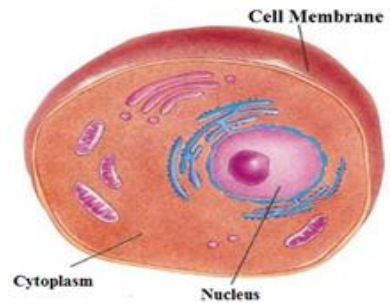
A

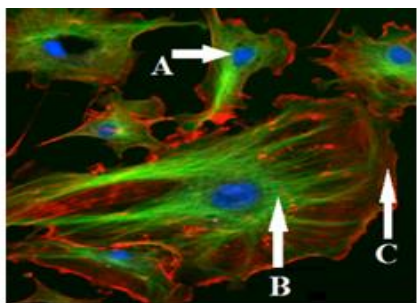

B
Fig. 2. (A) The main cell structure, (B) A specimen showing Endothelial cells stained with different dyes (Image courtesy: http://en.wikipedia.org/wiki/Fluorescence_microscope).

\section{REALTED WORKS}

\section{A. Cellular and Nuclear Image Segmentation Literature Background}

Semantic image segmentation in fluorescence microscopy analysis refers to the separation process of cell components from the surrounding background by finding the boundaries of cellular, nuclear or histological structures with an adequate accuracy from images of stained tissues with different markers, Fig. 2B. Nuclear segmentation is an important step in the pipeline of many cytometry analyses because it forms the basis of many other operations (e.g. cell counting, cell-cycle assignment, cell tracing) and is often the first step in the overall cell segmentation [22]. An increasing number of screenings and investigations are done using different types of fluorescence microscopy images either on individual or sequence (i.e. live-cells imaging) images.

In fluorescence labelled images of blood and bone marrow, high degrees of nuclei segmentation accuracy is reported by applying a classical image processing techniques such as shading correction and background (grayscale opening) followed by Otsu's method and watershed algorithm based on inverse distance transform [23]. In [24] a modified algorithm using the watershed algorithm based on morphological filtering operations is applied to choose the seeds of cell nuclei in tissue sections (i.e. foreground) and background as well. In this case, the merging of touching and overlapping regions is used to solve the over-seeded situations. In [24] method, it is required to manually choose and set specific values of certain parameters based on test images and then use them on images of the same dataset or images taken under the same conditions.

In [25], the problem of touching cells is addressed and treated by detecting the concave points from the polygonal approximations. After applying morphological filtering and adaptive thresholding to detect contour, this contours are segmented using the concave points. This approach is combined with a customized ellipse shape fitting such that each segment of the contour has a fitted ellipse.

A fully automated approach based on graph cut model is also used for segmenting the touching cell nuclei [26]. The background and the foreground separation is achieved using a minimal geodesic length, then the individual nuclei are found by a graph cut which include image gradient information and a priori knowledge about the shape of the nuclei. The graph-cut is also used for cells segmentation for the tracking problem in microscopy images [27].

The advantages of active contour method (flexibility), multi-resolution methods (low processing time), multi-scale methods (smoothing) and region-growing methods (statistical modelling) are combined in [28] to construct an accurate and fast cell nuclei segmentation algorithm.

A new method for leucocytes segmentation based on nuclei classification is presented in [29]. The overlapping and isolated configuration situations are classified based on Bayesian networks and stepwise merging strategy. Some morphological features of the nuclei, such as the compactness, smoothness and moments are used followed by a watershed algorithm to find the proper nuclei boundaries. The overlapping nuclei are segmented into isolated nuclei using an intensity gradient transform and watershed algorithm.

Some artificial neural network based approaches such as bidirectional associative memory (BAM) [9] is effectively used in medical segmentation application because it has some preferable features such as: supervision is required only for selecting texture primitives, no training is required and it is 
robust for the presence of noise and distortion. More reported segmentation algorithms in the field of fluorescence cell nuclei and histological structures can be found in [19]. In [30], a supervised learning-based system is proposed for segmenting different types of biomedical images where the focus was to describe a general purpose system that can, with few modifications, be used in a variety of image segmentation applications as long as enough labeled data is available for training. The system used the intensity neighborhoods as nonparametric feature vectors for pixel classification.

Although Fluorescence microscopy is a rapid expanding technique and it has made it possible to identify cellular components with a high degree of specificity, more attention is required to make its analysis fully automated and as meaningful as possible. Such work is challenging due to the large variations of features of the cellular components (size, shape, orientation, texture, etc.).

A totally automatic system is still not a reality; so much work remains, mainly in the early steps, which may involve segmentation, recognition of nuclei from the background followed by refinement, counting, and statistics calculations. From another side, many works still focus in certain regions (ROI) inside the image under processing rather than processing the whole image (i.e. inner and boundaries).

The main aim of this work and its further consequence modules is to focus on the specifics of images acquired by fluorescence microscopes (in particular, images cell nuclei) and design successful and efficient fully automated segmentation system that can be used to overcome these specifics simultaneously using tuned image processing and artificial intelligence techniques.

\section{B. Clinical Nuclei Related Work}

Many variations of the basic and CAD microscope instrument are now available and used in great success applications, allowing us to look into spaces much too small to be seen with the naked eye. The processing of digital microscope images includes the utilization of digital image processing technologies to analyze, model and visualize these images. Medicine, chemistry, cancer research, pharmacology, biological research and numerous related fields are common places for this type of microscope image processing. The processing of such images is improved by designing a direct special interfacing of microscope imaging instruments with image processing systems and interfaces (Fig. 1) [20], [21].

In many applications, it is very important to achieve accurate and efficient segmentation, classification and grouping of nuclei and cells in fluorescence microscopy images. This importance comes to enhance the understanding of cells functions [31] and enters the processing workflow of pathological diagnosis [32]. Examples of this include the immune-histochemically staining estimation and morphological grading where the detection of cell nuclei on histological slides is required.
From another hand, the precise quantitative statistics about nuclear structure and morphology along with their visualization can uncover important clues for the diagnosis of benign, pre-neoplastic, and neoplastic (cancer) lesions. Also, this type of quantification and classification should ease the understanding of the anatomical variation of different organs by the analysis of their corresponding tissues [33].

The nuclei segmentation were used as a basis to investigate the subcellular localization of proteins at a proteome-wide scale [34]. The cell nuclei were considered as seeds to perform protein segmentation using the watershed method and hence be able to identify the subcellular localization patterns even of complex ones.

\section{Dataset}

In this paper, the publicly available U2OS dataset described in [22] will be considered. Fig. 3 shows three images from the datasets under consideration. Although these images depict different levels of segmentation difficulty, most of them follow a similar histogram general profile where double-peaks (bimodal) are clear. It can be distinguished that in many occasions clustered touching nuclei are exist. Also, it is easy to figure out that the histograms of these images do not occupy the full dynamic range of the gray scale (i.e. 0-255) and they are concentrated on the low (dark) side of the scale. Different issues such as touching objects also takes place; these issues will be considered in the design of the different stages of the proposed segmentation system as will be described later.

\section{PROPOSED MethodOlOGY}

The main outline of the proposed methodology can be summarized as follow (Fig. 4) Stage-1) Preprocessing. Stage2) Detecting the nuclei candidates using NN, Stage-3) Classification of candidates into single isolated nucleus or clustered (overlapped or touching) nuclei, Stage-4) Separation of individual nucleus within clusters using modified watershed algorithm combined with NN iteratively, and 5) Refining each separated nucleus. The following subsections describe the details of these stages, followed by qualitative and quantitative evaluations.
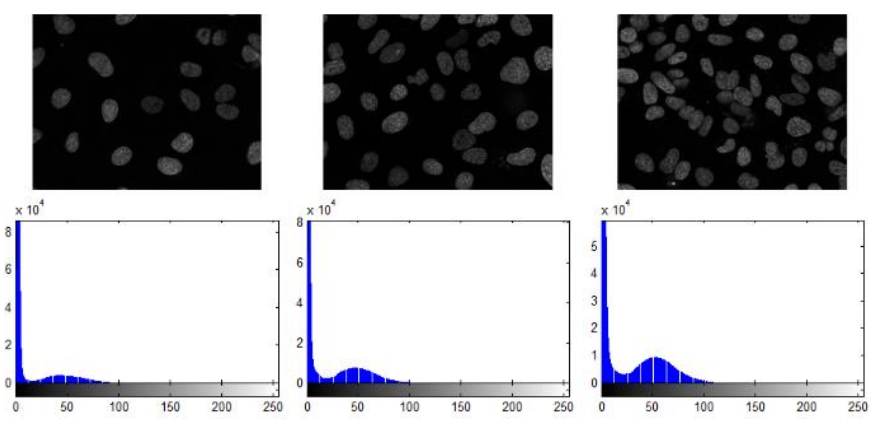

Fig. 3. (Top) Three examples of different cell nuclei images. (Left) dna-20, well separated with low number of nuclei, (Middle) dna-19, moderate level. (Right) dna-41, difficult clustered nuclei [22]. (Bottom) The corresponding histograms. 


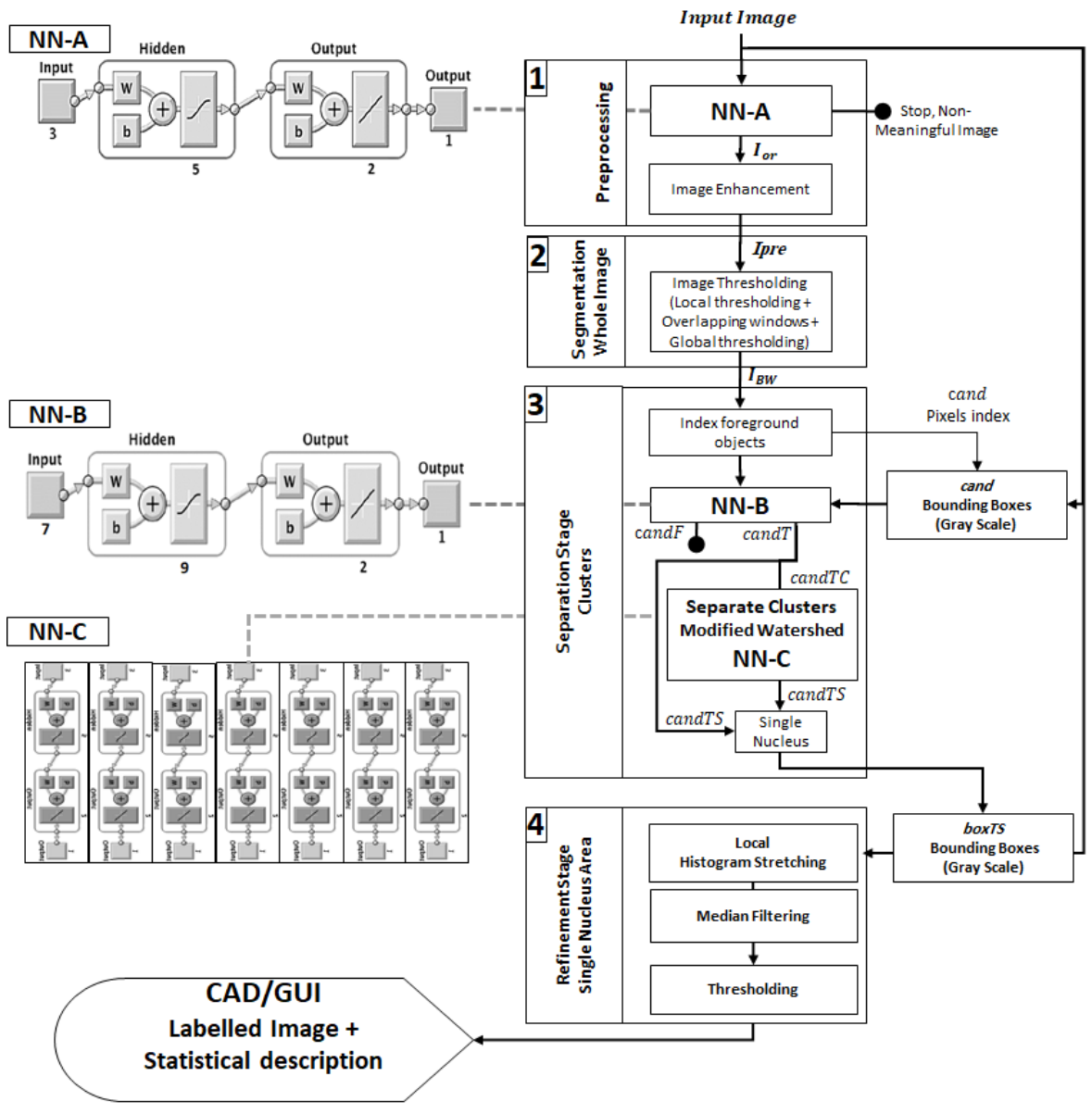

Fig. 4. The proposed cell nuclei segmentation system showing the multi stages of detection and verification.

\section{A. Preprocessing Stage}

Some input images contain no meaningful visual data, hence no nuclei can be identified or some noisy bright areas may be wrongly identified as nuclei. In the preprocessing stage, a neural network (NN-A) with back propagation algorithm (BPA) is used to identify this type of images. Part of the images were manually investigated and each image is assigned a flag ( $\mathrm{F} 1$, for meaningful images; F2, for nonmeaningful images). Then, three statistical features [(1)-(3)] were calculated for each image. The NN-A structure is: three inputs nodes, one hidden layer of 5 nodes and two output nodes. The training vector of NN-A is $[\mu, \sigma, E]$ and the neuron for output layer indicates $\mathrm{F} 1$ or $\mathrm{F} 2$ cases.

$$
\begin{aligned}
\mu & =\frac{\sum_{r=1}^{R} \sum_{c=1}^{C} I M_{r, c}}{R * C} \\
\sigma & =\sqrt{\frac{\sum_{r=1}^{R} \sum_{c=1}^{C}\left(I M_{r, c}-\mu\right)^{2}}{(R * C)-1}} \\
E & =-\sum_{n=0}^{255} p_{n} \log _{2} p_{n}
\end{aligned}
$$

where $\mu, \sigma, E$ are the mean, standard deviation and entropy of the input image (IM), respectively. $\mathrm{R}$ and $\mathrm{C}$ are the number of rows and columns of IM, respectively, and $p$ contains the 255-bins histogram count of IM. It is found that NN-A can exclude all non-meaningful images. This step will be useful in the generalization of the proposed method for other types of medical images.

Original image (hereinafter, $\boldsymbol{I}_{\boldsymbol{o r}}$ ) that has high information content is then preprocessed to reduce the noise and enhance the quality. Although the nuclei in such images are usually brighter than the dark background of the surrounding tissue, it is usually difficult to identify nuclei consistently over the whole image because some of these images are subject to nonuniform illumination and noise. To smooth out the possibility of uneven illumination, a gray morphological top-hat operator is applied.

$$
I_{\text {un }}=I_{\text {or }}-\left(\left(I_{\text {or }} \ominus S t r E l\right) \oplus \operatorname{StrEl}\right)
$$

where $\ominus$ and $\oplus$ denote erosion and dilation, respectively and $S t r E l$ is the structure element used to perform the opening operation. It is found that the optimum size of StrEl is correlated to the mean value of the image (the greater the area of nuclei in the image, the lesser the background area and hence the mean is greater).

Then, a common adaptive histogram stretching approach is applied on $I_{u n}$ to enhance the image contrast. In this approach, (18 pixels $\times 18$ pixels) sub images are processed individually to achieve contrast stretching of $95 \%$ (centered) of the sub image histogram, then the processed sub images are combined 
through a bilinear interpolation to compensate for the possible induced boundaries between these processed portions. Then a $9 \times 9$ median filter is applied to compensate for the increase in the noise amplitude which comes as a result of the above enhancement steps [1]. The result of this step is hereinafter called $\left(\boldsymbol{I}_{\text {pre }}\right)$, an example is shown in Fig. 8B.

\section{B. Segmentation Stage}

Generally, segmentation process can be achieved based on the separation of regions that have similar properties, such as gray level, color, texture, brightness, contrast, etc. In this stage, an adaptive gray level based thresholding approach is employed as follows:

1) Calculate $\boldsymbol{T} \boldsymbol{H}_{\boldsymbol{G}}$; the Otsu's [35] threshold of the global preprocessed image ( $\left.\boldsymbol{I}_{\text {pre }}\right)$.

2) Divide $\boldsymbol{I}_{\text {pre }}$ into square blocks (64 pixels $\times 64$ pixels) containing the gray levels $\left(\boldsymbol{B} \boldsymbol{L} \boldsymbol{K}_{\boldsymbol{g}}\right)$.

3) For each block $i\left(\boldsymbol{B} \boldsymbol{L} \boldsymbol{K}_{\boldsymbol{g} \boldsymbol{i}}\right)$, do the following:

a) Calculate

$$
T H_{a v g}=w . T H_{G}+(1-w) . T H_{L}
$$

where $\boldsymbol{T} \boldsymbol{H}_{\boldsymbol{L} \boldsymbol{i}}$ is the Otsu's threshold of $\boldsymbol{B} \boldsymbol{L} \boldsymbol{K} \boldsymbol{g}_{\boldsymbol{i}}$. And $\boldsymbol{w}$ is weighting factor, it is found experimentally that $\mathrm{W}=.85$ is an optimum choice.

b) Convert each block $\boldsymbol{B} \boldsymbol{L} \boldsymbol{K} \boldsymbol{g}_{\boldsymbol{i}}$ individually to a binary block (BLK $\boldsymbol{K}_{\boldsymbol{B} \boldsymbol{W} \boldsymbol{i}}$ ) using $\boldsymbol{T} \boldsymbol{H}_{\boldsymbol{a v g}}$ threshold calculated in step 0.

c) Combine the resulted binary blocks together to form the whole black-white image $\boldsymbol{I}_{\boldsymbol{B} \boldsymbol{W} \mathbf{1}}$.

4) Repeat steps 2) and 3) above using a block size of (32 pixels $\times 32$ pixels), this ends up with $\boldsymbol{I}_{\boldsymbol{B} \boldsymbol{W} \mathbf{2}}$.

5) Calculate $\boldsymbol{I}_{\boldsymbol{B} \boldsymbol{W}}=\boldsymbol{I}_{\boldsymbol{B} \boldsymbol{W} \mathbf{1}} \cdot \boldsymbol{I}_{\boldsymbol{B} \boldsymbol{W} \mathbf{2}}$; the bitwise logical Anding operation.

6) A series of morphological opening (using a disk shaped structure elements of 2, 3, 4 pixels radius) are performed on $\boldsymbol{I}_{\boldsymbol{B} \boldsymbol{W}}$ image to refine the shapes of the detected objects and remove noisy pixels and tiny white objects. Also, this operation removes small objects (i.e. dark pixels) inside the foreground of an image.

7) Then, a morphological flood filling operation is performed to remove small holes in the foreground.

\section{Single Nucleus and Clustered Nuclei Separation}

In this stage, the foreground objects in the binary $\boldsymbol{I}_{\boldsymbol{B} \boldsymbol{W}}$ image are isolated and indexed (labelled) individually, each object forms a region of possible nuclei candidates (cand). Some cand regions contain single nucleus, some regions contain nuclei cluster. These two types are called (candT). From the other hand, some regions contain faulty cand (candF) (i.e. Noise detected as nucleus), (Fig. 5).

\section{Non-nucleus Candidates Pruning}

It is recognized that some gray intensity properties of candF regions are not highly changeable. As an example, it is found by deep investigation that the Otsu's threshold of nuclei blocks (i.e. blocks contains true nuclei) is almost close to the mean of the pixels within the block, while non-nuclei blocks don't satisfy this. Also, for the nuclei blocks, it is found that Kurtosis (peakedness) [36] of the part of the histogram right to the Otsu's threshold is higher than that of non-nuclei blocks, Fig. 6.

To prune candF, a NN (hereinafter NN-B) with BPA training is used. Several NN topologies were designed and tested to determine the optimum NN-B. It is found that the optimum NN-B contains (7 input nodes, one hidden layer with 9 nodes, 2 output nodes).

A number of (candF) and (candT) regions (similar to Fig. 5) were manually cropped. Then, the features described in (6)-(12) were calculated for these regions. The vector [ $\mu_{R}, \sigma_{R}$, skew $_{R}$, kurtosis $_{R}, D R_{R}, B P, R O B_{R}$ ] after normalization represent the input training vector to NN-B. The neuron of the output layer indicates whether the block is (candF) or (candT).

$$
\begin{aligned}
& \mu_{R}=\frac{\sum_{r=1}^{R_{R}} \sum_{C=1}^{C_{R}} \operatorname{cand}_{r, c}}{R_{R} * C_{R}} \\
& \sigma_{R}=\sqrt{\frac{\sum_{r=1}^{R_{R}} \sum_{c=1}^{C_{R}}\left(\operatorname{cand}_{r, c}-\mu_{R}\right)^{2}}{\left(R_{R} * C_{R}\right)-1}} \\
& \text { skew }_{R}=\frac{1}{R_{R} * C_{R}} \sum_{r=1}^{R_{R}} \sum_{c=1}^{C_{R}}\left(\frac{\operatorname{cand}_{r, c}-\mu_{R}}{\sigma_{R}}\right)^{3} \\
& \text { kurtosis }_{R}=\frac{\sum_{r=1}^{R_{R}} \sum_{c=1}^{C_{R}}\left(\operatorname{cand}_{r, c}-\mu_{R}\right)^{4}}{R_{R} * C_{R} * \sigma_{R}{ }^{4}}-3 \\
& D R_{R}=\max _{r, c} \operatorname{cand}_{r, c}-\min _{r, c} \operatorname{cand}_{r, c} \\
& B P_{R}=\frac{\forall\left(\text { cand }_{r, c} \geq 1.25 * \mu_{R}\right), \sum_{r=1}^{R_{R}} \sum_{C=1}^{C_{R}}(1)}{R * C} \\
& R O B_{R}=\frac{B P}{R_{R} * C_{R}}
\end{aligned}
$$

where $\mu_{R}, \sigma R$, skew $_{R}$, kurtosis $_{R}$ are the mean, standard deviation, skew, kurtosis of the cropped region (candR), respectively. $R_{R}$ and $C_{R}$ are the number of rows and columns of candR, respectively. $D R_{R}$ represent the dynamic range of intensities of candR, $B P$ represents the number of bright pixels in candR nprmalized to the full image size, and $R O B_{R}$ represents the ratio of bright pixels to the total area of candR. $R$ and $C$ as defined in (1).

By training and testing, it is found that NN-B is capable to prune candF and accept candT for further processes with higher degree of accuracy. Numerical evaluation of the NN-B goodness is included in section IV.

\section{E. Separation of Clustered Nuclei}

Some candT contain more than one nuclei, this is caused by touching or overlapping nuclei Fig. 5-B. To get accurate count and statistics of nuclei, it is important to split candT regions that contain clustered nuclei into single nucleus (candTS). To achieve this, a region based segmentation using watershed transform is applied on candT. The idea behind the basic watershed transform is to define the catchment basins and the watershed lines between them. Generally, the watershed transform is applied to the image gradient. 


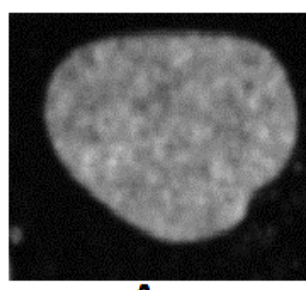

A

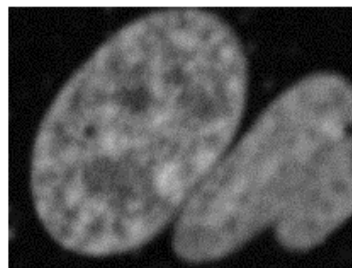

B

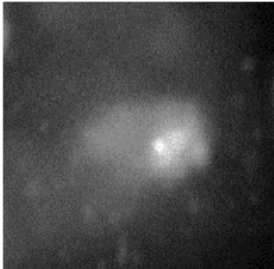

C

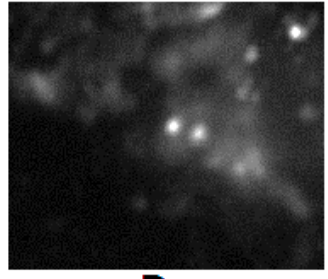

D

Fig. 5. Samples of different regions, (A) single nucleus (candT), (B) clustered touching nuclei (candT), (C and D) non-nuclei (candF).
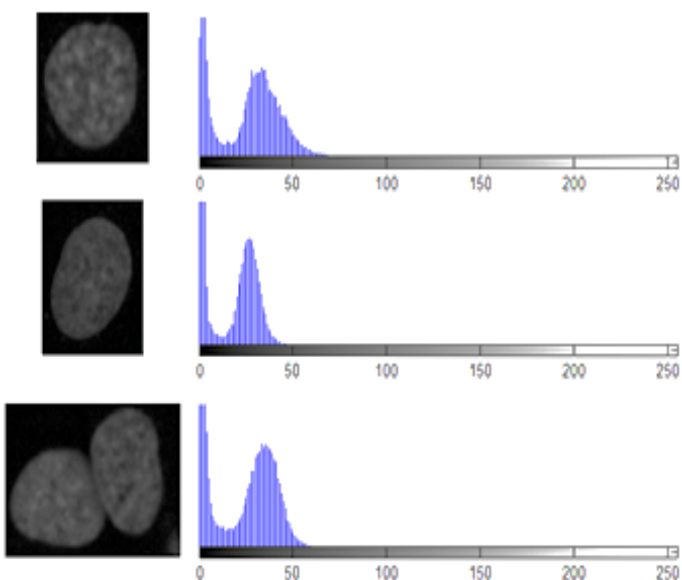
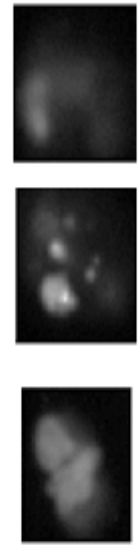
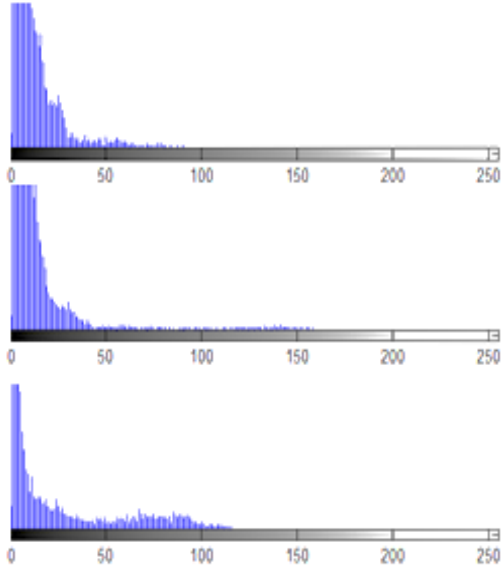

Fig. 6. Samples of different sub regions: (Left) nuclei and their corresponding histograms (right) non-nuclei region and their corresponding histograms.

Direct application of the watershed transform results in a large number of tiny regions. To solve this problem, the proposed system presents a modified iterative watershed algorithm to separate the touching nuclei in nuclei clusters. The iterative scenario is controlled and tuned using a neural network (hereinafter NN-C). To control the number of separated nuclei within each candT, candT is scaled using a variable scaling parameter (SP). The separation algorithms are as follows:

a) Initiate SP to 1. (all watershed regions are detected).

b) Define ScandT; the scaled version of candT using SP.

c) Apply watershed transform on ScandT

d) Calculate the areas of the detected regions in step $\mathrm{C}$, and choose the 10 largest $\left(\operatorname{Reg}_{i} ; i \in\{1: 10\}\right)$.

e) For each $\operatorname{Reg}_{i}$, using (13)-(19), calculate the following features' vector:

$$
\begin{aligned}
& F V_{i} \\
& =\left[\text { Area }_{i}, \operatorname{Per}_{i}, \text { Ratio }_{i}, \operatorname{CenX}_{i}, \operatorname{CenY}_{i}, \operatorname{MinAx}_{i}, \operatorname{Max}_{i} x_{i}\right]
\end{aligned}
$$

f) For each $\operatorname{Reg}_{i}$, use the trained NN-C to estimate another version of $F V_{i}$ (called $F V_{i N N C}$ )

g) Calculate the squared error

(SErr) between $F V_{i}$ and $F V_{i N N C}$.

h) If SErr is reducing, then reduce SP linearly and perform a new iteration starting from step B. If the reduction in SErr is below a predefined threshold, then stop iterations and choose the regions candTS that have small SErr less than a predefined threshold that were calculated during training.

$$
\text { Area }_{i}=\frac{A_{i}}{R_{i} * C_{i}}
$$

$$
\begin{aligned}
& \operatorname{Per}_{i}=\frac{P_{i}}{R_{i} * C_{i}} \\
& \text { Ratio } i_{i}=\frac{\operatorname{Per}_{i}}{\text { Area }_{i}} \\
& \operatorname{CenX}_{i}=\frac{\sum_{j=1}^{A_{i}} X_{i j}}{R_{i} * A_{i}} \\
& \operatorname{CenY}_{i}=\frac{\sum_{j=1}^{A_{i}} Y_{i j}}{C_{i} * A_{i}} \\
& \operatorname{MinAx}_{i}=\text { length of minor axis of } \operatorname{Reg}_{i} \\
& \operatorname{MaxAx}_{i}=\text { length of major axis of } \operatorname{Reg}_{i}
\end{aligned}
$$

where $A_{i}$ and $P_{i}$ are the number of pixels in the area and in the perimeter of the region i, respectively. Area $_{i}$, Per $_{i}$, CenX $_{i}$ and $\operatorname{Cen} Y_{i}$ are the area, perimeter, center of mass in horizontal direction and center of mass in vertical direction, respectively.

$F V_{i N N C}$ vector represents the neuron of the output layer of the NN-C. candT and ScandT are normalized to a fixed dimension $(\mathrm{Rn} * \mathrm{Cn})$ and the mean value for each row and the mean value for each column of both candT and ScandT are calculated and saved. These constructed vector have a length $(2 * \mathrm{Rn}+2 * \mathrm{Cn})$ represent the input training and testing vector for NN-C. Fig. 7. shows examples' results of the separation step.

\section{F. Refinement Stage}

In this stage, every single nuclei candTS is processed alone to refine its borders. This stage will take out local nonnuclei pixels that were misclassified as nuclei members. From the other hand, the local nuclei pixels that were misclassified as non-nuclei will be added to their corresponding nucleus. This is done as follows: 


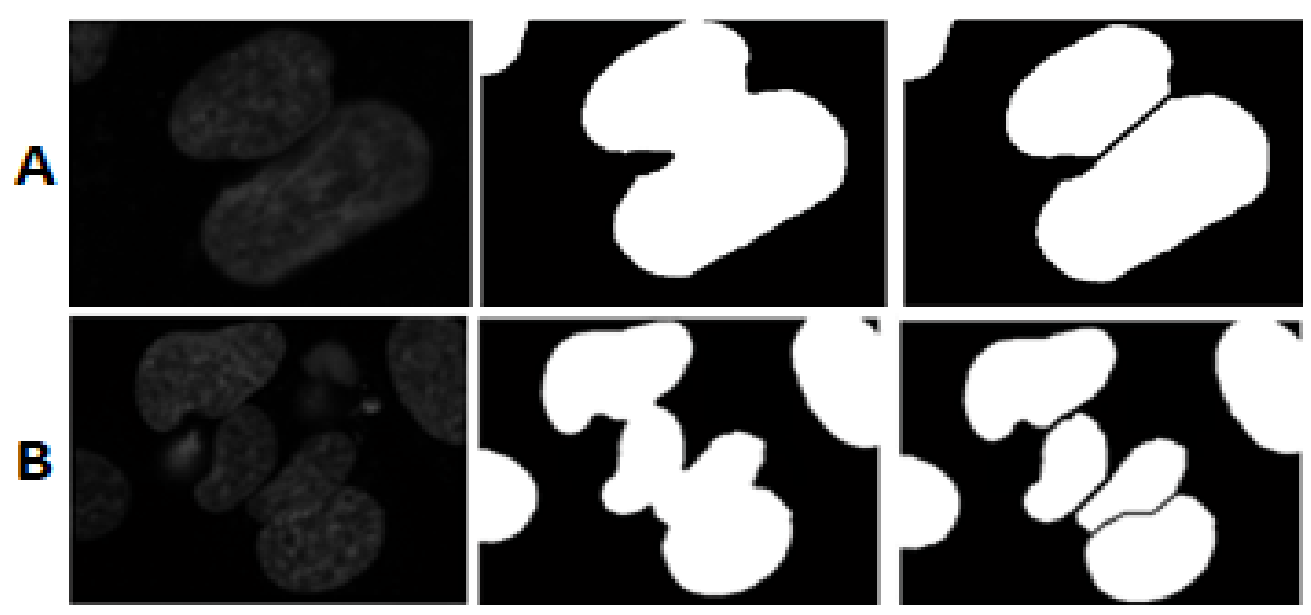

Fig. 7. Two examples of Separation of clustered nuclei. (Left) Enhanced cropped regions. (Center) Initial segmentation (CandT). (Right) Split nucleus (CandTS) using modified watershed and NN-C.
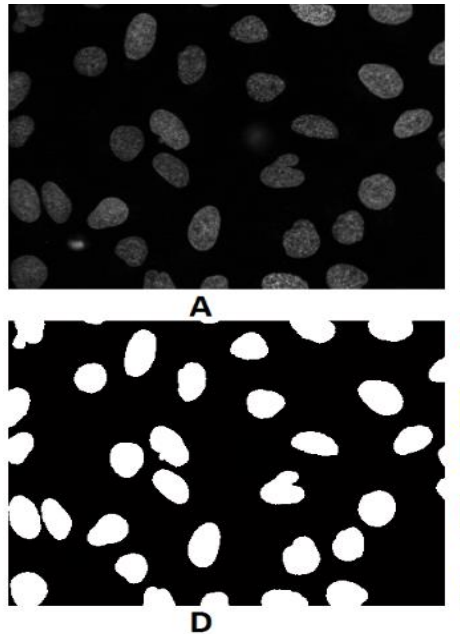
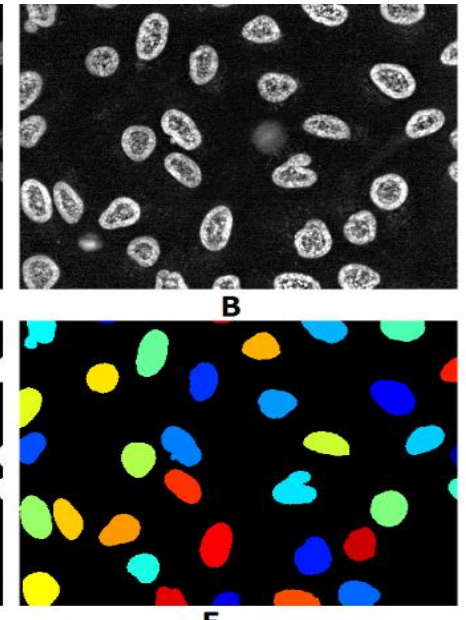

E
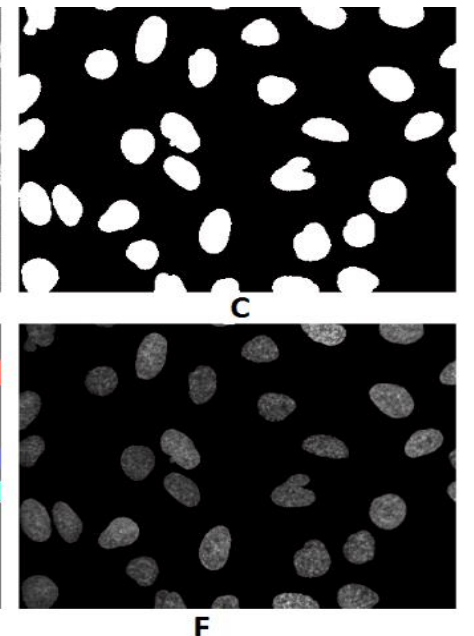

Fig. 8. (A) Input image. (B) Enhanced image. (C) Segmented image. (D) Refined result. (E) Color labeling. (F) Original gray scale profile of the segmented nuclei.

a) Calculate the centroid of the candTS

b) Define the bounding rectangular box boxTS that contains all the pixels of candTS and 10\% more pixels from each side (Top, Bottom, Lift, and Right).

c) From the original image $\boldsymbol{I}_{\text {or }}$; crop out the sub image $\boldsymbol{I}_{\boldsymbol{o r}} \boldsymbol{b o x}$ contained within the boundaries of boxTS.

d) Enhance $\boldsymbol{I}_{\boldsymbol{o r}} \boldsymbol{b o x}$ individually by applying a histogram stretching transform over $95 \%$ of the range and a median filter of size $8 * 8$. This step will increase the independency of each nuclei boundary.

e) Use the calculated centroid in step 1 as a seed to start a region growing based thresholding within boxTS. In this step, the region growing is not allowed to expand in the directions where other candTS are located. This vital to avoid rejoining of separated nuclei.

It is found that this enhancement stage highly improves the local region of each individual nuclei and hence provide more fine and accurate details of the nuclei borders.

An intensive empirical study based on several cross validation runs were carried out on different nuclei images of different noise levels and different segmentation difficulty to choose some features' values in the above stages. Some parameters were fixed and some of them are automatically changing (tuned) according to other statistics calculated during the processing of each individual image. No assumptions were assumed and no human interaction is required to choose certain areas in the image under processing (i.e. the proposed system operates on the whole image rather than a specific region of interest $\mathrm{ROI}$ ).

Along with the proposed system, an interactive graphical user interface (GUI) has been developed. This GUI allows the user to select the data set and then all the associated images are displayed. Fig. 9. shows the developed GUI including all the display options.

\section{PERFORMANCE EVALUATION AND DISCUSSION}

For evaluation, the proposed methodology is applied on the (U20S) dataset described above. In order to evaluate the NN-B performance, the false acceptance rate (FDR) is calculated. FDR is an error measure that shows the probability that a candF is detected as a candT. Samples of the calculated FDR for randomly selected images are shown in Table I. 
Row 3 shows the FDR without using the NN-B, while row 4 shows the FDR using the NN-B. It is apparent that the FDR is reduced using the NN-B. Using NN-B reduces the average FDR from $12.8 \%$ to $2.1 \%$.

Fig. 10 shows examples of the segmentation obtained by the proposed methodology compared to the provided handlabelled (ground truth) images. For finer details comparison, a zoomed version for some of the nuclei shown in Fig. 10. are also shown in Fig. 11.

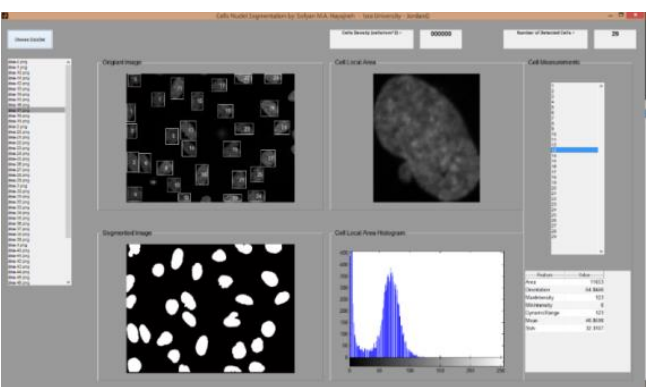

Fig. 9. The proposed GUI.

TABLE I. FDR FOR RANDOMLY SELECTED IMAGES WITH AND WITHOUT USING NN-B

\begin{tabular}{|l|l|l|l|l|l|l|l|l|l|l|l|l|l|l|l|l|}
\hline Image number (dna-) & 0 & 10 & 14 & 18 & 23 & 29 & 3 & 30 & 35 & 39 & 4 & 40 & 45 & 48 & 8 & \\
\hline Number of candT & 41 & 42 & 47 & 44 & 43 & 37 & 28 & 33 & 29 & 35 & 27 & 34 & 28 & 32 & 28 & Avg. \\
\hline $\begin{array}{l}\text { FDR } \\
\text { (No NN-B) (\%) }\end{array}$ & 4.90 & 4.80 & 8.50 & 20.5 & 4.70 & 8.10 & 21.4 & 9.10 & 20.7 & 14.3 & 11.1 & 17.6 & 3.60 & 6.30 & 21.4 & $\mathbf{1 2 . 8}$ \\
\hline $\begin{array}{l}\text { FDR } \\
\text { (NN-B) (\%) }\end{array}$ & 0 & 0 & 2.1 & 2.3 & 0 & 2.7 & 3.6 & 0 & 6.9 & 2.9 & 0 & 2.9 & 0 & 3.1 & 0 & $\mathbf{2 . 1}$ \\
\hline
\end{tabular}
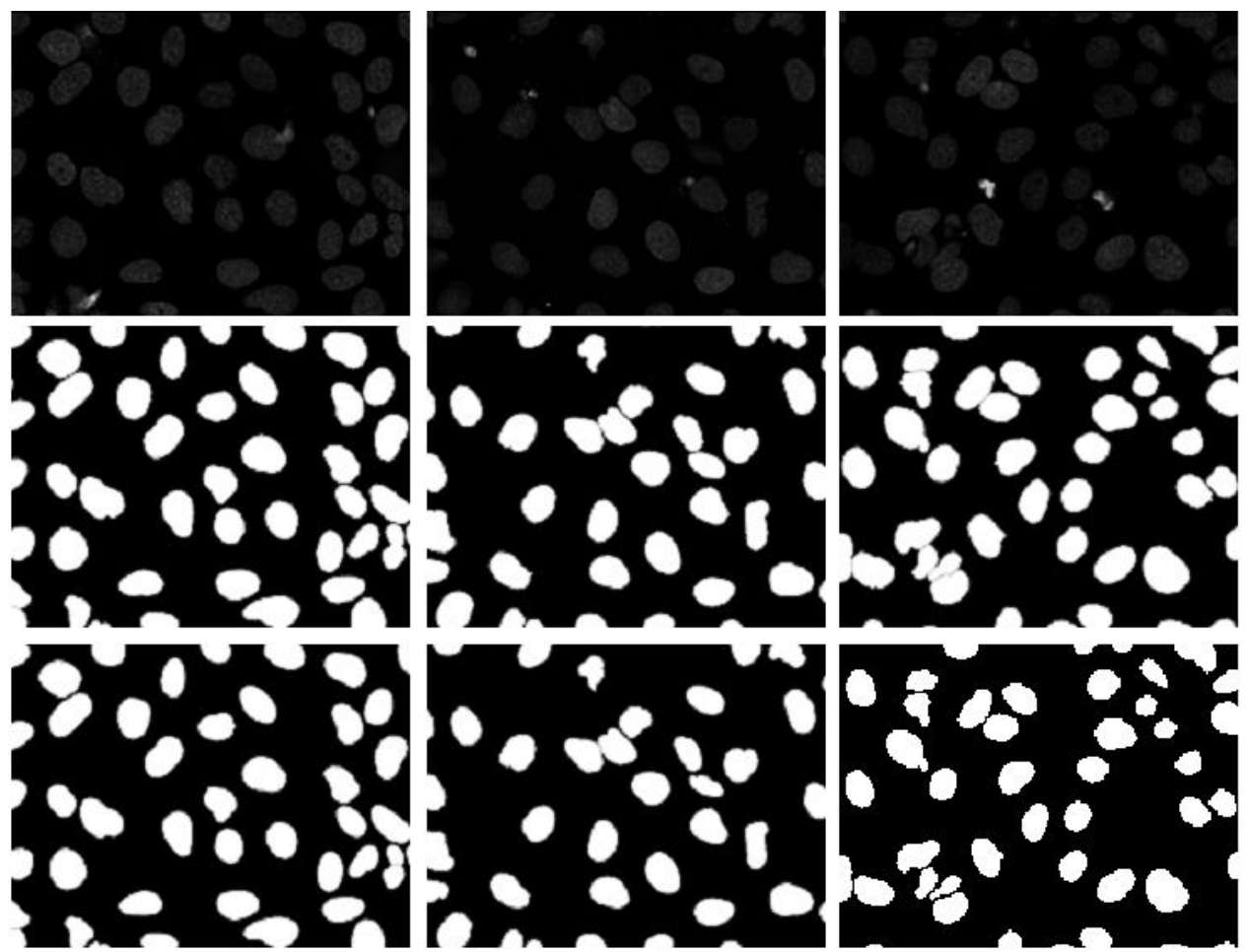

Fig. 10. Three examples of nuclei segmentation. (top) original, (middle) ground truth, (bottom) proposed

Almost, as Fig. 11 depicts, it is found that ground truth nuclei borders are bigger (outer) than the segmented ones. It is thought that this is due to an oversize segmentation in the hand labelling. Anyway, a simple morphological dilation process could overcome this issue. However, we purposely prefer not to apply this post processing in the refinement stage since the main focus is to describe and evaluate the proposed method. Fig. 12 depicts this fact clearly, it is clear that the total area of all segmented nuclei - using the proposed method - in each image is less than that in the ground truth reference images. This is ensures that a simple dilation process could lead to more area convergence. Again, we purposively prefer not to apply this step.

Although Fig. 10 and 11 show a very qualitative reasonable result, quantitative evaluation should provide another proof of satisfaction. To this end, the proposed method is compared to other methods in literature [22] that were applied on the (U20S) dataset.

Let (IP) represents the proposed binary image output and (IGT) represents the ground truth binary image, [22] described different quantitative performance metrics that include: (1) Hausdroff (HD) metric and normalized sum of distances 
(NSD), both can be considered as a spatially based metrics, (2) (Split) error: two IP nuclei are assigned to a single IGT nucleus, (Merge): single IP nucleus embody two IGT nuclei, (Add): an IP nucleus is assigned to the IGT background, and (Miss): an IGT nucleus is assigned to the IP background. Table II shows the result of the proposed methodology compared to other methods.

As stated above, the proposed method provides slight less area in most of the cases. However, this does not affect the error count metrics as they depend on counting objects numbers and comparing pairs of objects (i.e. one to one object in IP and IGT). From the other hand, this slightly affects the values of the HD and NSD as they are aware of the spatial locations of nuclei and their contours so that good values of HD and NSD metrics can still apparently be obtained.

For the same reason, we got lower Dice metric value (0.88), this value can reach an average of 0.94 when a dilation operation is applied on the final segmented binary nuclei. Again, we purposively prefer not apply such preprocessing step.

In general, using the watershed approach leads to less merge errors while increasing the split error [22]. In the proposed algorithm, it is apparent (Table II) that we can achieve less merge errors using the watershed while keeping split errors at low levels. This is due to applying a modified region growing algorithms that can merge regions that are wrongly separated (split) in the watershed step.

Also, a reasonable (add error) metric were obtained due to applying the non-nucleus pruning operation based on an NN training. From the other hand, the (miss error) were kept at low levels due to applying an adaptive thresholding criteria that takes into account the local and global intensity variations through the image.

It is important to compare the computational cost of the proposed method to some previous methods. It can be shown that the proposed method presents a low computational approach while providing reasonable results. In average, the proposed method takes $\sim 23$ seconds to label the input image. This represents a significant reduction in computational cost compared to many other methods that include [30] which takes 49.2 minutes using a supervised learning approach, while in [41] it takes 30 minutes using the template matching approach.

TABLE II. Quantitative Comparison of the Proposed Method and Other Methods. Each Value Represents an AVERAGed PeRformance METRIC OVER ALL THE IMAGES IN THE DATASET

\begin{tabular}{|c|c|c|c|c|c|c|c|c|}
\hline \multirow{2}{*}{ Method } & \multirow{2}{*}{ Reference } & \multirow{2}{*}{$\begin{array}{l}\text { Pixel } \\
\text { Dice }\end{array}$} & \multicolumn{2}{|c|}{ Spatially Based } & \multicolumn{4}{|c|}{ Error Count } \\
\hline & & & HD & NSD & Split & Merge & Add & Miss \\
\hline Otsu's & [35] & 0.88 & 30.6 & 0.11 & 1.10 & 2.40 & 0.3 & 5.60 \\
\hline $\begin{array}{l}\text { RC) } \\
\text { Iterative Thresholding }\end{array}$ & [37] & 0.92 & 34.8 & 0.12 & 1.10 & 2.40 & 0.3 & 5.50 \\
\hline Watershed (direct) & [24] & 0.82 & 25.9 & 0.34 & 13.2 & 1.20 & 1.8 & 3.20 \\
\hline Watershed (gradient) & {$[38]$} & 0.85 & 34.6 & 0.30 & 7.70 & 2.00 & 2.0 & 2.90 \\
\hline Merging & [39] & 0.91 & 13.1 & 0.07 & 1.80 & 2.10 & 1.0 & 3.30 \\
\hline Active Mask & [40] & - & 148.3 & 0.55 & 10.50 & 2.10 & 0.4 & 10.8 \\
\hline Template matching & [41] & - & 77.8 & 0.06 & 0.58 & 1.45 & 0.9 & 3.48 \\
\hline Level set & {$[42]$} & - & 96.6 & 0.09 & 1.10 & 0.35 & 2.75 & 0.85 \\
\hline K-means & [43] & - & 94.6 & 0.11 & 1.56 & 0.30 & 2.6 & 1.60 \\
\hline Proposed & & 0.88 & 23.3 & 0.07 & 0.46 & 0.30 & 0.35 & 1.10 \\
\hline
\end{tabular}
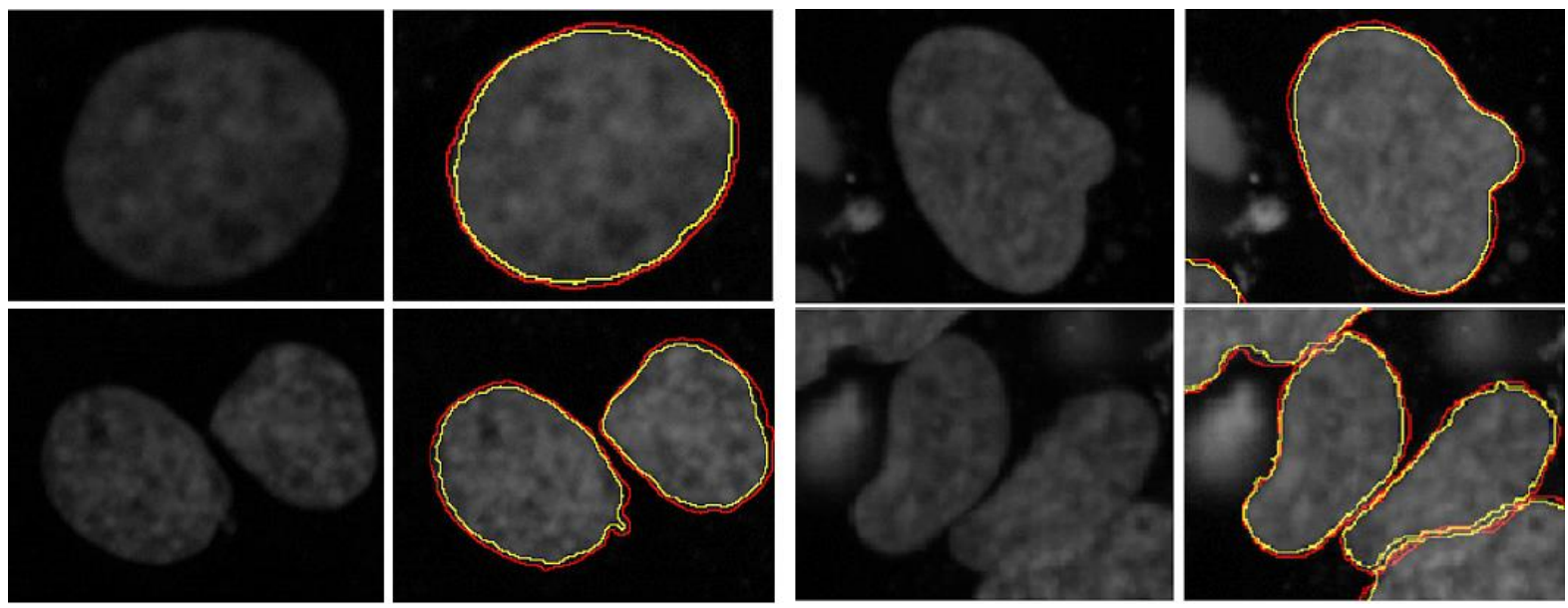

Fig. 11. Zoomed versions of Fig. 10. (left) original (right-red) ground truth (right-yellow) proposed. 


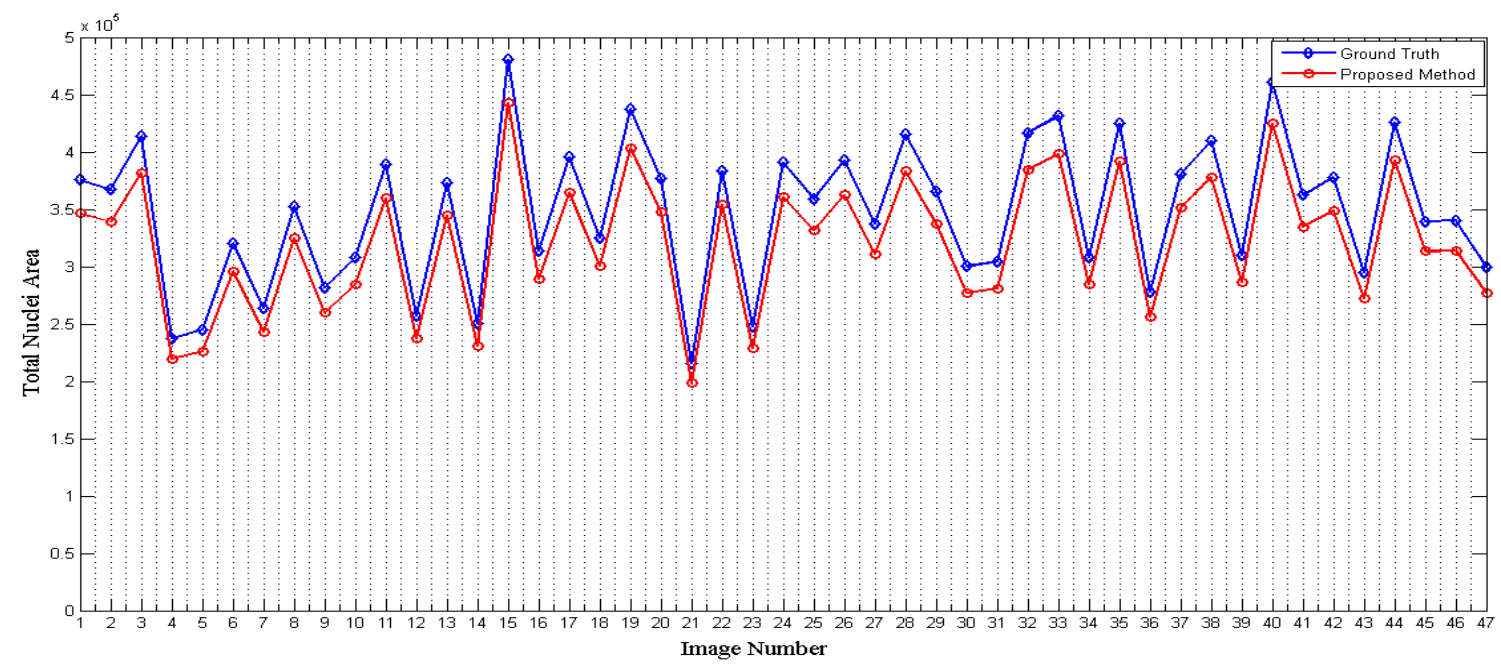

Fig. 12. Total area of nuclei detected in both ground truth reference images and the proposed methodology.

Good computational time cost of 15 seconds were achieved by [31], but using a (Linux system of 48GB RAM). It is still thought that the proposed algorithm dramatically outperforms this time because a (Windows system of $2 \mathrm{G}$ RAM) is used compared to 48GB RAM.

\section{CONCLUSIONS AND FURTHER WORK}

The proposed system in this work combined many image processing techniques in a single automated platform to automate processing of microscopy cell images. Different stages of the proposed system are supported by cascaded neural networks used to extract features and tune other processes. A GUI is also designed and provided to make the proposed system more user friendly and helpful in the forthcoming works. It was shown that the proposed system is capable of providing reasonable and very good promising results in the segmentation stages. It is hoped that the successful of this work and its subsequent development will pave the way for our vision of advanced levels of processing that includes real time processing of living cells and nuclei, and the three dimensional modelling of cells and histological structures. Future work to improve the outcome of the current work should include more accurate and efficient techniques for improving the nuclei segmentation. Another types of analysis such as automated cell detection, counting, classification, and tracking could then be built into a toolbox that would facilitate automation analysis of stem cell behavior as an example.

The proposed system is designed to deal with the whole image region without focusing into certain (ROI) regions which means more accurate and meaningful results. The proposed system depends on auto tuning of some related parameters which means that it can be extended to be applied on other datasets without the need for new methodologies. The proposed system shows a promising results compared to other systems. It also shows a rescannable reduction in processing time which makes it applicable in near real time diagnosis systems.

The ground truth images of the database under consideration are hand-segmented to separate touching nuclei without labelling overlapped regions. So that, the separation stage described in the methodology section is designed to get accurate count of nuclei without taking into account the full area of each nucleus. As future work, it is hoped to enhance the refinement stage by increasing the accuracy of separation of clustered nuclei so that overlapped region is associated more accurately and more meaningfully to the separated nuclei. From the other hand, it is of the future planes to provide such system as a software as a service (SaaS) [44] and to allow the integration of this system with other related systems.

\section{REFERENCES}

[1] R. C. Gonzalez and R. E. Woods, Digital image processing, 2002.

[2] M. J. Aschwanden, "Image processing techniques and feature recognition in solar physics," Solar Physics, vol. 262, pp. 235-275, 2010.

[3] M. Sonka, V. Hlavac, and R. Boyle, Image processing, analysis, and machine vision: Cengage Learning, 2014.

[4] C. Solomon and T. Breckon, Fundamentals of Digital Image Processing: A practical approach with examples in Matlab: John Wiley \& Sons, 2011.

[5] T. W. Nattkemper, "Automatic segmentation of digital micrographs: A survey," Medical Informatics, vol. 11, pp. 847-851, 2004.

[6] M. M. Fraz, P. Remagnino, A. Hoppe, B. Uyyanonvara, A. R. Rudnicka, C. G. Owen, and S. A. Barman, "Blood vessel segmentation methodologies in retinal images-A survey," Computer methods and programs in biomedicine, vol. 108, pp. 407-433, 2012.

[7] S. Wienert, D. Heim, K. Saeger, A. Stenzinger, M. Beil, P. Hufnagl, M. Dietel, C. Denkert, and F. Klauschen, "Detection and Segmentation of Cell Nuclei in Virtual Microscopy Images: A Minimum-Model Approach," Sci. Rep., vol. 2, 07/11/online 2012.

[8] N. Sharma and L. M. Aggarwal, "Automated medical image segmentation techniques," Journal of medical physics/Association of Medical Physicists of India, vol. 35, p. 3, 2010.

[9] N. Sharma, A. K. Ray, S. Sharma, K. K. Shukla, S. Pradhan, and L. M. Aggarwal, "Segmentation and classification of medical images using texture-primitive features: Application of BAM-type artificial neural network," Journal of Medical Physics / Association of Medical Physicists of India, vol. 33, pp. 119-126, 2008.

[10] M. J. McAuliffe, F. M. Lalonde, D. McGarry, W. Gandler, K. Csaky, and B. L. Trus, "Medical image processing, analysis and visualization in clinical research," in Computer-Based Medical Systems, 2001. CBMS 2001. Proceedings. 14th IEEE Symposium on, 2001, pp. 381-386.

[11] I. Bankman, Handbook of medical image processing and analysis: academic press, 2008. 
[12] W. Birkfellner, Applied medical image processing: a basic course: Taylor \& Francis, 2014.

[13] J. Peti-Peterdi and P. D. Bell, "Confocal and two-photon microscopy," in Renal Disease, ed: Springer, 2003, pp. 129-138.

[14] F. Gorunescu, "Data mining techniques in computer-aided diagnosis: non-invasive cancer detection," Journal of Gastrointestinal and liver diseases, vol. 16, pp. 427-430, 2007.

[15] S. Kara and F. Dirgenali, "A system to diagnose atherosclerosis via wavelet transforms, principal component analysis and artificial neural networks," Expert Systems with Applications, vol. 32, pp. 632-640, 2007.

[16] M. S. Sharif, R. Qahwaji, S. Hayajneh, S. Ipson, R. Alzubaidi, and A. Brahma, "An efficient system for preprocessing confocal corneal images for subsequent analysis," in Computational Intelligence (UKCI), 2014 14th UK Workshop on, 2014, pp. 1-8.

[17] B. Salah, M. Alshraideh, R. Beidas, and F. Hayajneh, "Skin cancer recognition by using a neuro-fuzzy system," Cancer informatics, vol. 10, p. 1, 2011.

[18] K. Doi, "Computer-aided diagnosis in medical imaging: historical review, current status and future potential," Computerized medical imaging and graphics, vol. 31, pp. 198-211, 2007.

[19] C. SMOCHINĂ, P. HERGHElEgIU, and V. MANTA, "Image Processing Techniques used in Microscopic Image Segmentation," Gheorghe Asachi Technical University of Iaşi.2011.

[20] Q. Wu, F. Merchant, and K. Castleman, Microscope image processing: Academic press, 2010.

[21] H. Lodish, A. Berk, S. L. Zipursky, P. Matsudaira, D. Baltimore, and J. Darnell, Microscopy and Cell Architecture. New York: W.H. Freeman \& Company, 2000.

[22] L. P. Coelho, A. Shariff, and R. F. Murphy, "Nuclear segmentation in microscope cell images: a hand-segmented dataset and comparison of algorithms," in Biomedical Imaging: From Nano to Macro, 2009. ISBI'09. IEEE International Symposium on, 2009, pp. 518-521.

[23] V.-T. Ta, O. Lézoray, A. Elmoataz, and S. Schüpp, "Graph-based tools for microscopic cellular image segmentation," Pattern Recognition, vol. 42, pp. 1113-1125, 2009.

[24] C. Wählby, I. M. SINTORN, F. Erlandsson, G. Borgefors, and E. Bengtsson, "Combining intensity, edge and shape information for 2D and 3D segmentation of cell nuclei in tissue sections," Journal of Microscopy, vol. 215, pp. 67-76, 2004.

[25] X. Bai, C. Sun, and F. Zhou, "Splitting touching cells based on concave points and ellipse fitting," Pattern recognition, vol. 42, pp. 2434-2446, 2009.

[26] O. Daněk, P. Matula, C. Ortiz-de-Solórzano, A. Muñoz-Barrutia, M. Maška, and M. Kozubek, "Segmentation of touching cell nuclei using a two-stage graph cut model," in Image Analysis, ed: Springer, 2009, pp. 410-419.

[27] H. F. Yang and Y. Choe, "Cell tracking and segmentation in electron microscopy images using graph cuts," in IEEE International Symposium on Biomedical Imaging: From Nano to Macro, 2009. ISBI'09., 2009, pp. 306-309.

[28] G. Srinivasa, M. C. Fickus, Y. Guo, A. D. Linstedt, and J. Kovacevic, "Active mask segmentation of fluorescence microscope images," Image
Processing, IEEE Transactions on, vol. 18, pp. 1817-1829, 2009.

[29] M. R. JEONG, B. Ko, and J. Y. NAM, "Overlapping nuclei segmentation based on Bayesian networks and stepwise merging strategy," Journal of microscopy, vol. 235, pp. 188-198, 2009.

[30] C. Chen, J. A. Ozolek, W. Wang, and G. K. Rohde, "A general system for automatic biomedical image segmentation using intensity neighborhoods," Journal of Biomedical Imaging, vol. 2011, p. 8, 2011.

[31] J.-P. Bergeest and K. Rohr, "Efficient globally optimal segmentation of cells in fluorescence microscopy images using level sets and convex energy functionals," Medical Image Analysis, vol. 16, pp. 1436-1444, $10 / / 2012$.

[32] T. J. Fuchs and J. M. Buhmann, "Computational pathology: Challenges and promises for tissue analysis," Computerized Medical Imaging and Graphics, vol. 35, pp. 515-530, 10// 2011.

[33] W. Wei, J. A. Ozolek, Slepc, x030C, D. ev, A. B. Lee, C. Cheng, and G. K. Rohde, "An Optimal Transportation Approach for Nuclear StructureBased Pathology," Medical Imaging, IEEE Transactions on, vol. 30, pp. 621-631, 2011.

[34] L. P. Coelho, T. Peng, and R. F. Murphy, "Quantifying the distribution of probes between subcellular locations using unsupervised pattern unmixing," Bioinformatics, vol. 26, pp. i7-i12, 2010.

[35] N. Otsu, "A Threshold Selection Method from Gray-Level Histograms," IEEE Transactions on Systems, Man and Cybernetics, vol. 9, pp. 62-66, 1979.

[36] J. S. Suri, Advances in diagnostic and therapeutic ultrasound imaging: Artech House, 2008.

[37] T. Ridler and S. Calvard, "Picture thresholding using an iterative selection method," IEEE transactions on Systems, Man and Cybernetics, vol. 8, pp. 630-632, 1978.

[38] L. Vincent and P. Soille, "Watersheds in digital spaces: an efficient algorithm based on immersion simulations," IEEE Transactions on Pattern Analysis \& Machine Intelligence, pp. 583-598, 1991.

[39] G. Lin, U. Adiga, K. Olson, J. F. Guzowski, C. A. Barnes, and B. Roysam, "A hybrid 3D watershed algorithm incorporating gradient cues and object models for automatic segmentation of nuclei in confocal image stacks," Cytometry Part A, vol. 56, pp. 23-36, 2003.

[40] G. Srinivasa, M. Fickus, M. N. G. Rivero, S. Y. Hsieh, Y. Guo, A. D. Linstedt, and J. Kovacevic, "Active mask segmentation for the cellvolume computation and Golgi-body segmentation of HeLa cell images," in Biomedical Imaging: From Nano to Macro, 2008. ISBI 2008. 5th IEEE International Symposium on, 2008, pp. 348-351.

[41] C. Chen, W. Wang, J. A. Ozolek, and G. K. Rohde, "A flexible and robust approach for segmenting cell nuclei from 2D microscopy images using supervised learning and template matching," Cytometry Part A, vol. 83, pp. 495-507, 2013.

[42] T. F. Chan and L. Vese, "Active contours without edges," Image processing, IEEE transactions on, vol. 10, pp. 266-277, 2001.

[43] K. S. Ravichandran and B. Ananthi, "Color skin segmentation using Kmeans cluster," February 2220162009.

[44] S. Hayajneh, "Cloud Computing SaaS Paradigm for Efficient Modelling of Solar Features and Activities," International Journal of Cloud Applications and Computing (IJCAC), vol. 5, pp. 20-34, 2015. 